\title{
Albumin-Binding Domain Conjugate for Near-Infrared Fluorescence Lymphatic Imaging
}

Cynthia A. Davies-Venn, ${ }^{1,3}$ Bonnie Angermiller, ${ }^{1}$ Nathaniel Wilganowski, ${ }^{1}$ Pradip Ghosh, ${ }^{1}$ Barrett R. Harvey, ${ }^{2}$ Grace Wu, ${ }^{1}$ Sunkuk Kwon, ${ }^{1}$ Melissa B. Aldrich, ${ }^{1}$ Eva M. Sevick-Muraca ${ }^{1}$

${ }^{1}$ The Center for Molecular Imaging, The Brown Foundation Institute of Molecular Medicine, University of Texas Health Science Center, 1825 Pressler Street, SRB 330A, Houston, TX 77030, USA

${ }^{2}$ The Center for Immunology and Autoimmune Diseases, Brown Foundation Institute of Molecular Medicine, University of Texas Health Science Center, 1825 Pressler St. SRB 330A, Houston, TX 77030, USA

${ }^{3}$ Translational Biology and Molecular Medicine Program, Baylor College of Medicine, One Baylor Plaza, Houston, TX 77030, USA

\begin{abstract}
Purpose: The aim of this study was to develop and characterize a novel peptide imaging agent for noninvasive near-infrared fluorescence imaging of protein transport by the lymphatics. An imaging agent consisting of a cyclic albumin-binding domain (CABD) peptide, with sequence, Arg-Leu-Ile-Glu-Asp-Ile-Cys-Leu-Pro-Arg-Trp-Gly-Cys-Leu-Trp-Glu-Asp-Asp-Lys, was conjugated to a near-infrared fluorophore, IRDye800CW, allowing for enhanced vascular uptake, retention, and fluorescence imaging.

Procedure: Characterization of the CABD-IRDye800 peptide conjugate was performed using fluorescence spectroscopy to assess optical properties and SDS-PAGE and Biacore binding assays to determine binding affinity and specificity. Fluorescence imaging of normal C57BL/6 mice was conducted to monitor lymphatic uptake and retention.

Results: CABD-IRDye800 exhibited approximately six times greater fluorescent yield and greater stability than indocyanine green, an agent previously used in humans to image lymphatic vasculature. The agent exhibited affinity for albumin with $\mathrm{IC}_{50}$ and $\mathrm{Kd}$ in the nanomolar range and demonstrated superior retention characteristics within mouse lymphatics when compared with IRDye800CW.

Conclusions: CABD-IRDye800 has utility for assessing lymphatic function in mouse models of human lymphatic disease and the potential for use in clinical diagnostic imaging of the lymphatic vasculature.

Key words: Near-infrared fluorescence, Lymphatic imaging, Albumin peptide conjugate

List of Abbreviations ABD Albumin-binding domain; ANOVA Analysis of variance; AAALAC Association for Assessment and Accreditation of Laboratory Animal Care; CABD Cyclic albuminbinding domain; CLAMC Center for Laboratory Animal Medicine and Care; EMCCD Electronmultiplying charge-coupled device; Fab Antibody binding fragment; HSA Human serum albumin; ICG Indocyanine green; MSA Mouse serum albumin; NHS-EDC $N$-Hydroxysuccinimide- $N$-ethyl- $N$ (dimethylaminopropyl)-carbodiimide; NIR Near infrared; NP Nanoparticle; OVA Ovalbumin; ROI Region of interest; SA Serum albumin; ScDb Single-chain diabody; SDS-PAGE Sodium dodecyl sulfate-polyacrilamide gel electrophoresis; SPR Surface plasmon resonance; MW Molecular weight
\end{abstract}

Electronic supplementary material The online version of this article (doi:10.1007/s11307-011-0499-x) contains supplementary material, which is available to authorized users.

Correspondence to: Eva Sevick-Muraca; e-mail: eva.sevick@uth.tmc.edu

\section{Introduction}

lthough the lymphatic system was first described in the 1600 s and is recognized to be important to human 
health $[1,2]$, much remains to be learned about its structure and function in both health and disease. Functions of the lymphatics include immune regulation, protein transport, and fluid homeostasis, which are carried out by a complex network of vessels. The network begins with the initial lymphatic capillaries found in the dermis that drain into larger lymphatic vessels which in turn, propel lymph to lymph nodes through the action of lymphangions, and ends at the subclavian vein where lymph is returned to the blood circulation. Failure of lymphatic vessels to function properly due to a genetic defect, injury, or drug interaction may lead to swelling and edema, that if not resolved, leads to lymphedema, a condition in which fluid retention in the extremities causes chronic edema and pain. Eventual fibrosis of the affected tissue may occur owing in part to the lack of lymphatic protein transport from interstitial tissues.

Human genetic studies have, thus far, revealed three genes linked to congenital lymphedema: VEGFR3 [3, 4], SOX18 [5], and FOXC2 [6]. Animal studies with conditional and total knockout of these and other genes [7-10] have shown they are important for early development, establishment, and maintenance of the lymphatic system. More recent work shows that acquired forms of lymphedema, such as that following cancer nodal staging surgeries, may also be associated with gene susceptibility in humans [11]. In addition, one of the most common off-target effects of marketed and investigational therapeutics is the edema that can occur due to the decreased transport capacity of the lymphatics, enhanced capillary filtration of the blood vasculature, or a combination of both.

Unfortunately, the lymphatic architecture and function in both humans and mouse models of disease has not been well characterized due to the lack of in vivo imaging techniques with sufficient temporal and spatial resolution and with adequate contrast agents (for review, see Ref. [12]). Currently, the clinical gold standard for lymphatic imaging is lymphoscintigraphy which consists of an intradermal or subcutaneous injection of a radiocolloid contrast agent, typically $99 \mathrm{mTc}$, followed by imaging with a gamma camera. The injection can be painful; imaging is time consuming requiring several minutes to acquire images; the procedure exposes the patient to ionizing radiation; and the images exhibit poor spatial and temporal resolution $[13,14]$. Due to these limitations, other imaging methods including computed tomography $[15,16]$ and magnetic resonance [17] have been investigated for enhanced sensitivity imaging of the lymphatics. Unfortunately, they require potentially painful and damaging cannulation of lymphatic vessels, large amounts of contrast agent, and extensive imaging equipment and instrumentation. Recently, our research team and others (for review, see Ref. [18]) have demonstrated clinical, noninvasive, near-infrared (NIR) fluorescence imaging of human lymphatics using the tricarbocynanine dye, indocyanine green (ICG), and comparatively compact NIR imaging instrumentation. NIR fluorescence with excitation at $>750 \mathrm{~nm}$ is advantageous for deep tissue imaging owing to a high signal-to-noise ratio, minimal light absorption, maximal tissue penetration, and low background due to minimal autofluorescence. ICG has maximal absorption at $\sim 780 \mathrm{~nm}$ and is approved for clinical use in vascular and hepatic function testing on the basis of its dark green color [19] and its ability to associate with albumin [20]. Upon properly designing instrumentation for sensitive collection of its fluorescence, we have performed investigational human lymphatic imaging with rapid image acquisition (200-800 ms) under conditions of ICG microdosing after intradermal injections of as little as $25 \mu \mathrm{g}$ [21-26]. Furthermore, lymphatic phenotyping of disease on the basis of vessel architecture and function has also been demonstrated using this method [25].

Although ICG is retained in the lymphatics due to its association with serum proteins, it is an unstable fluorophore requiring its use within hours of reconstitution in saline. A detailed study of degradation kinetics showed that under aqueous conditions, ICG degrades with first-order kinetics. Under the most favorable conditions of darkness and at low temperature $\left(8^{\circ} \mathrm{C}\right)$, the half life of ICG was approximately $20 \mathrm{~h}$ [27]. Exposure to light and increased temperature further accelerated the degradation of ICG. The intensity and wavelength of light also significantly impacted the degradation time. Interestingly, ICG exposure to light at $786 \mathrm{~nm}$, (which is the wavelength used by our camera systems), caused faster degradation and a minimal half life of only $2.3 \mathrm{~h}$ [27]. An additional HPLC degradation study of ICG in saline showed that by $25 \mathrm{~h}$ a $100 \mu \mathrm{g} / \mathrm{mL}$ solution of ICG had a $70 \%$ degradation peak with only $30 \%$ of the ICG peak remaining (Electronic Supplementary Materials). The rapid appearance of degradation products makes ICG less favorable as a clinically acceptable fluorophore than newly available, more stable fluorophores. These alterations of the fluorescent properties of ICG result in a dim signal, and impair the visualization of deep lymphatic structures such as the saphenous lymphatic channel of the lower leg. Increasing the concentration of ICG leads to a gradual decline in fluorescence intensity [27], which arises due to stacking of dye molecules and resultant quenching which has been described previously [28, 29].

Herein, we present our work to develop a brighter, but low molecular weight NIR fluorescent conjugate that is capable of deep tissue lymphatic imaging while also associating with plasma proteins for retention within vascular spaces. The use of plasma proteins such as albumin has recently become a popular strategy for improving the pharmacokinetics and decreasing the clearance of therapeutics targeted to a variety of diseases including arthritis [30], HIV [31], and many types of cancer [32-36]. These strategies often take advantage of the fact that due to increased metabolic activity and enhanced permeability of the blood vasculature of tumors and inflammatory tissue, there is an increased uptake and accumulation of serum proteins in these tissues. Further- 
more, a lack or impairment of lymphatic vasculature around the tumor may cause a further increase of protein. Application of albumin binding strategies has shown efficacy in vitro and also in several animal models, for summary see Table 1 [37-50]. Half-life extension methods have included the development of a maleimide derivative of doxorubicin conjugated to albumin, which covalently binds to the free thiol group of cysteine-34 on the albumin protein. As cysteine- 34 is unique to albumin, this protein marker provides a general strategy for increasing retention time of therapeutic conjugates of maleimide derivatives by their direct binding to endogenous albumin [37]. While the endogenous albumin strategy is primarily useful for maleimides, after investigating several half-life extension strategies including pegylation, $N$-glycosylation, and HSA fusion, Linhult and Johansson [38, 39], identified an albumin-binding domain (ABD) from the bacterial protein Streptococcal G that was able to bind with nanomolar to fentomolar affinity for albumin. They conjugated this ABD to antibody fragments including a Fab and single-chain diabody $(\mathrm{scDb})$. The scDb-ABD was found to have a 10 -fold increased half life and higher accumulation in CEA-positive tumors and be favorable for tumor therapy while significantly reducing molecular weight compared with direct antibody fusion methods. In a related approach, in 2002 Dennis et al. [40] used phage display to isolate several peptides that bound with high affinity to serum albumin of multiple species and which could potentially be conjugated to a variety of ligands using $\mathrm{N}$ hydroxysuccinimide (NHS) ester chemistry. Mutational analysis and binding studies showed that several serum albumin (SA) peptides were capable of binding with nanomolar affinity and upon conjugation to a Fab fragment were able to extend plasma half-life 25-58-fold thereby increasing therapeutic efficacy. Specifically, one peptide, SA21 showed a high affinity for albumin at a site distinct from albumin's other important ligand binding sites and stability in serum while also maintaining the ability of the Fab to bind tissue factor in vivo. Thus, this approach provides a novel method of association to albumin that can be useful for enhancing vascular retention.

We have employed a cyclic version of SA21, and we term the cyclic albumin-binding domain (cABD) peptide. We have conjugated the stable, cABD peptide, with a bright NIR fluorophore, IRDye $800 \mathrm{CW}$, which itself has favorable clearance, safety, and toxicity profiles [51]. The chemical structure of cABD-IRDye 800 is shown in Fig. 1. We have also characterized and demonstrated the superiority of cABD-IRDye 800 compared with ICG in studies of mouse lymphatics. The use of cABDIRDye 800 as a NIR imaging agent will enable a better understanding of lymphatic vasculature, help elucidate the pathophysiology of lymphatic disease, and provide insight into other diseases in which the lymphatic system may play a role.

\section{Materials and Methods}

\section{Agent Preparation}

ICG was obtained from Akorn (Lake Forest, IL), stored at room temperature and reconstituted in sterile phosphate-buffered saline (PBS) immediately prior to use. IRDye ${ }^{\circledR} 800 \mathrm{CW}$ NHS ester was purchased from LI-COR Biosciences (Lincoln, NE) and reconstituted in PBS or dimethyl sulfoxide (DMSO) prior to use. The cABD peptide, RLIEDICLPRWGCLWEDDK, with disulfide bond between Cys 7 and Cys13, >95\% purity, was obtained from Bachem (Torrance, CA), stored as received at $-20^{\circ} \mathrm{C}$, and reconstituted immediately before use in Sorenson's phosphate buffer ( $\mathrm{pH} 8-8.5$ ). cABD peptide conjugation to IRDye800 was performed by combining equimolar amounts of IRDye800 in DMSO and cABD peptide in phosphate buffer. The specific conjugation reaction occurs by formation of a stable amide bond between the free amine on the terminal lysine of the cABD peptide and the NHS ester reactive group on IRDye 800 . Although it may appear there are two potential reactive sites on the peptide: (1) the terminal lysine and (2) the terminal NH2 group; the terminal $\mathrm{NH} 2$ on the peptide is an amide, and is therefore, unreactive (Fig. 1). Conjugation occurred overnight at $4{ }^{\circ} \mathrm{C}$ on a rotator platform under dark conditions. The conjugated cABD peptide was successfully purified using a semi-prep HPLC system equipped with a Zorbax column (C-18, 250× $10 \mathrm{~mm}$ ) using a mobile phase of $0.1 \%$ TFA in $\mathrm{H}_{2} \mathrm{O} / 0.1 \%$ TFA in $\mathrm{CH}_{3} \mathrm{CN}$ gradient and flow rate of $1 \mathrm{~mL} / \mathrm{min}$. A retention time of 32.3 min was measured using EzChrome Elite software. The conjugate was then dried using a vacuum evaporator (Thermo Scientific Inc.) to remove water and residual TFA and stored at $-20^{\circ} \mathrm{C}$ protected from light.

\section{Characterization of Fluorophores}

Fluorescence optical properties of the fluorophores were determined using the Fluorolog Tau-3 Spectrofluorometer (Horiba Jobin Yvon, Edison, NJ) and absorbance was recorded using the DU-800 Spectrophotometer (Beckman Coulter, Brea CA). All measurements were taken at fluorophore concentrations $\leq 1 \mu \mathrm{M}$. At this low concentration, confounding effects such as quenching of ICG are avoided and fluorophores are more easily compared. In addition, each fluorophore was purified by lyophilization to remove TFA or solvent, prior to being measured to the nearest microgram and prepared at the desired concentration by dilution in the appropriate solvent. Molecular weights (MW) for ICG and IRDye 800 were confirmed by the respective vendors and cABD-IRDye $800 \mathrm{MW}$ was confirmed by mass spectrometry. Consistency in the preparation methods for each fluorophore also helped ensure accuracy in the final concentration of each fluorophore used to record optical properties and in the event of any minor solvent or salt effects, these would be similar among all fluorophores. Excitation and emission spectra of $1 \mu \mathrm{M}$ solutions of ICG, IRDye 800 and cABD-IRDye 800 were obtained at wavelengths of 785 and $830 \mathrm{~nm}$ respectively, with an integration time of $0.3 \mathrm{~s}$. Extinction coefficients were determined from the slope of absorbance at $785 \mathrm{~nm}$ as a function of the concentration of serial dilutions of each agent at concentrations of $0.01,0.1,0.5$, 


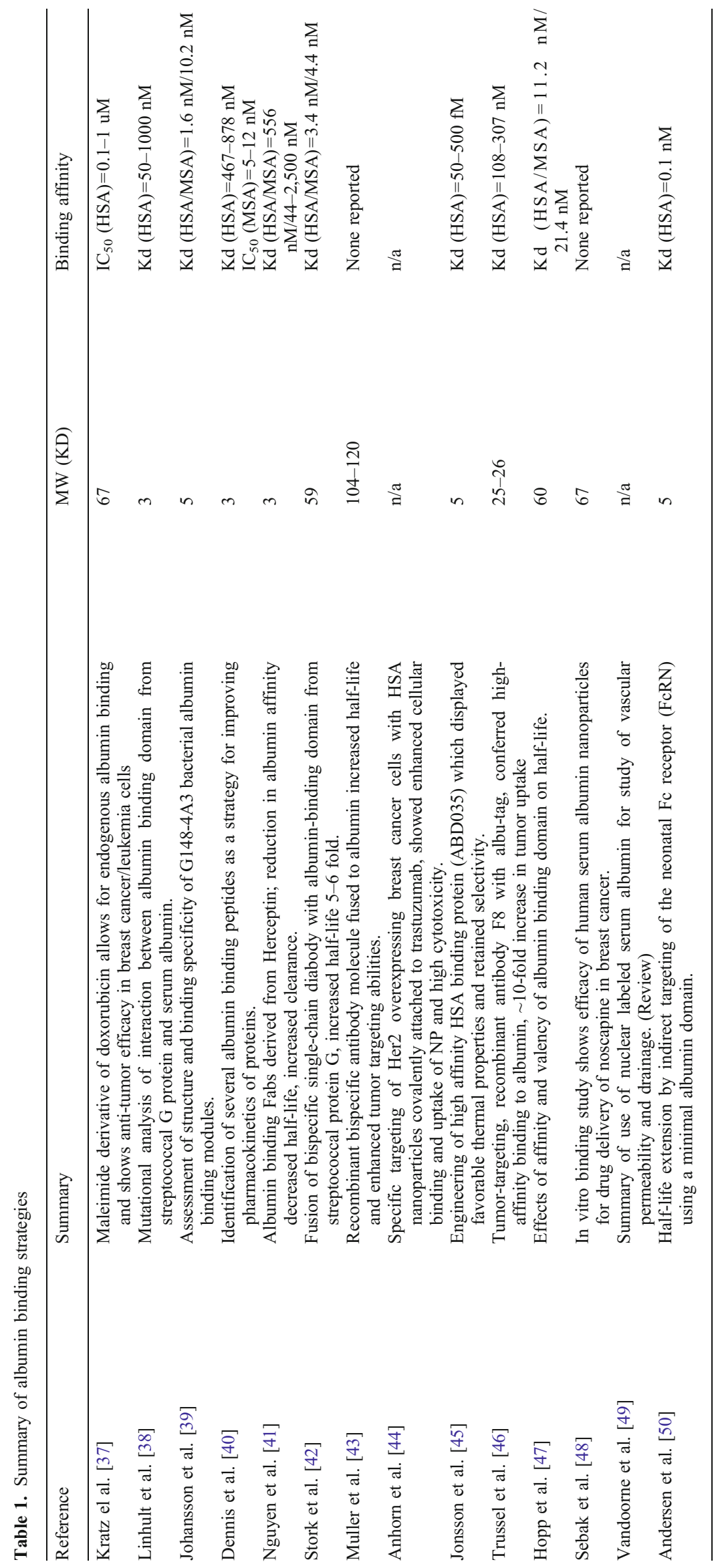




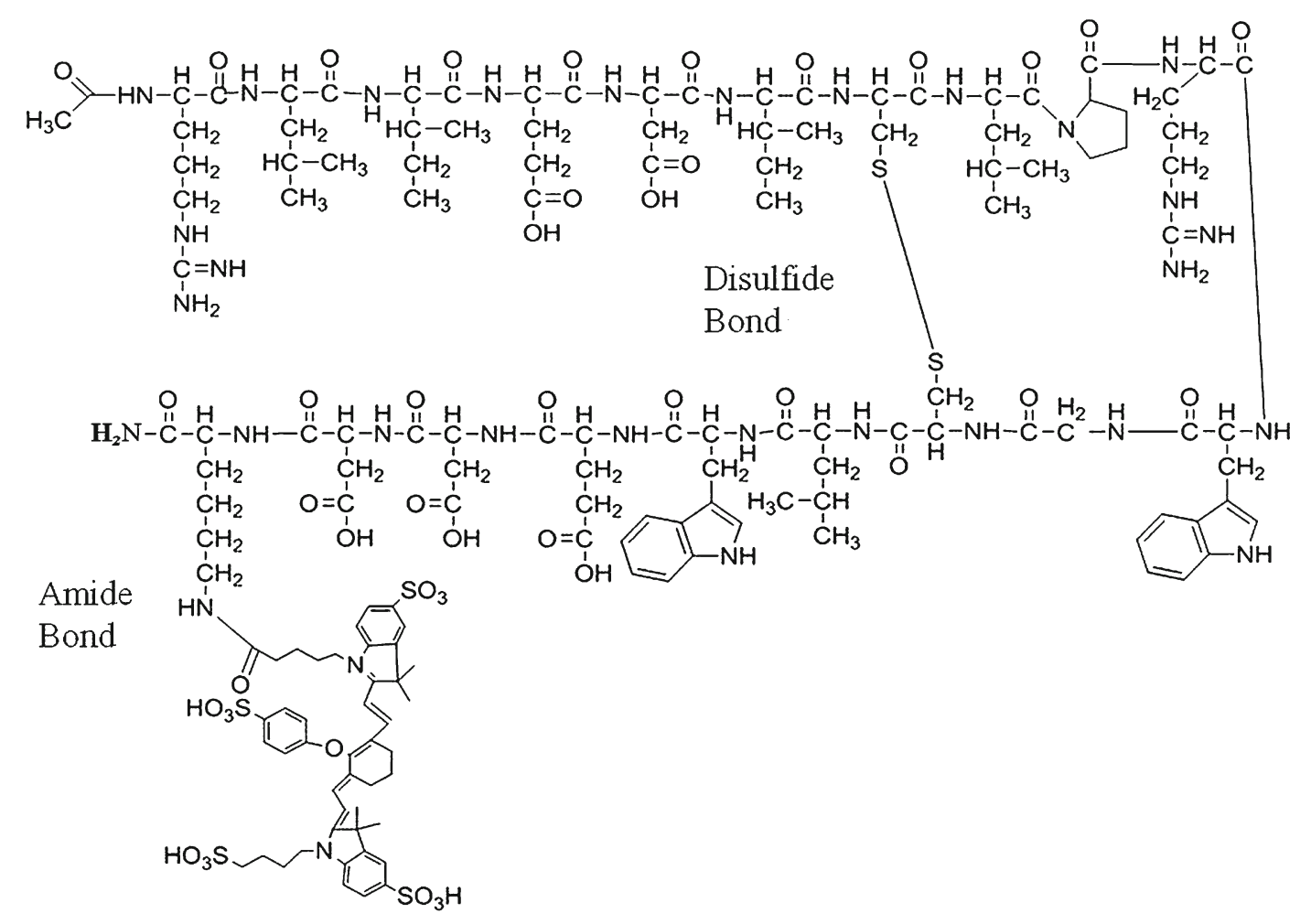

IRDye800

Fig. 1. A chemical structure diagram showing CABD-IRDye800 including the specific site of conjugation and formation of an amide bond between the free amine in the terminal lysine side chain of the CABD peptide and the NHS ester reactive site of IRDye800 CW. The S-S, disulfide bond was added to stabilize the peptide and maintain uniform product once conjugated to IRDye800.

and $1 \mu \mathrm{M}$. Fluorescence quantum yield was determined by the comparative method of Williams et al. [52] using the quantum yield of ICG (in water) at 785/830 $\mathrm{nm} \mathrm{[53]} \mathrm{as} \mathrm{a} \mathrm{reference} \mathrm{standard} \mathrm{in} \mathrm{which}$ fluorescence of each fluorophore at the above-mentioned concentrations was plotted as a function of absorbance. The gradient of this plot and that of the ICG reference standard was then used to calculate quantum yield using Williams' equation [52]. Frequency domain lifetime measurements were acquired using $1 \mu \mathrm{M}$ concentrations of each fluorophore over ten frequencies between 20 and $200 \mathrm{MHz}$, with an excitation and emission of 785 and $830 \mathrm{~nm}$, respectively, and integration time of $12 \mathrm{~s}$. ICG, with known lifetime $0.56 \mathrm{~ns}$ [53] was used as a reference standard.

Mass spectrometry was performed by the Mass Spectrometry Laboratory in the Pharmaceutical Development Center of the Department of Experimental Therapeutics at the University of Texas MD Anderson Cancer Center in Houston TX. The lyophilized cABD-IRDye800 conjugate was dissolved in 80:20 methanol $/ 0.2 \%$ formic acid aqueous $(\mathrm{pH} 3.0)$ at a concentration of $1 \mu \mathrm{g} / \mathrm{mL}$. This solution was directly infused into a Waters Quattro ZQ single quadrupole mass spectrometer, operated in electrospraypositive ionization and full scan mode. The confirmation of the molecular weight was done by application of deconvolution software, Waters MaxEnt 3.

An HPLC stability study was performed over 15 days under four storage conditions: water at room temperature or $4^{\circ} \mathrm{C}$ and PBS at room temperature or $4^{\circ} \mathrm{C}$. Stability was assessed by an analytical HPLC system equipped with a Hitachi LaChrom Ultra column $(\mathrm{C}-18,5 \mu \mathrm{m}, 4.6 \times 150 \mathrm{~mm})$ using a mobile phase of $0.1 \%$ TFA in $\mathrm{H}_{2} \mathrm{O} / 0.1 \%$ TFA in $\mathrm{CH}_{3} \mathrm{CN}$ gradient and flow rate of $1 \mathrm{~mL} / \mathrm{min}$ over $60 \mathrm{~min}$. The percent area of the conjugate peak at a retention time of $24.9 \mathrm{~min}$, Fig. 2, was used to determine the purity and stability of the cyclic conjugate. Stability was measured at day $0,1,2,3,4,7$, and 15 .

The $\mathrm{IC}_{50}$ of cABD-IRDye 800 was determined using a competitive binding assay modified from Dennis et al. [40]. Briefly, Maxisorp fluorescent plates (Nunc, Rochester, NY) were coated with mouse serum albumin (Sigma, St Louis, MO) and incubated overnight at $4^{\circ} \mathrm{C}$. Plates were washed with PBS+ $0.05 \%$ Tween 20 (Sigma, St. Louis, MO) to remove unbound albumin and were then blocked with TBS in casein (Pierce, Rockford, IL) for $1 \mathrm{~h}$ at $25^{\circ} \mathrm{C}$. Equimolar amounts of unconjugated peptide and peptide conjugate were combined in centrifuge tubes for five different concentrations $(0-50 \mathrm{nM})$ of cABD peptide. The resulting peptide solution was then plated on the albumin coated plate, covered, and incubated for $1-2 \mathrm{~h}$ at room temperature. The plate was washed with PBS with $0.05 \%$ Tween 20 to remove unbound peptide, followed by fluorescence imaging at $800 \mathrm{~nm}$ on an Odyssey ${ }^{\circledR}$ Infrared Imager (LI-COR ${ }^{\circledR}$ Biosciences, Lincoln, NE). The average and standard deviation of four $\mathrm{IC}_{50}$ values were determined.

Affinity measurements were obtained via SPR using a BIACORE T100 (GE Healthcare) instrument similar to a work 


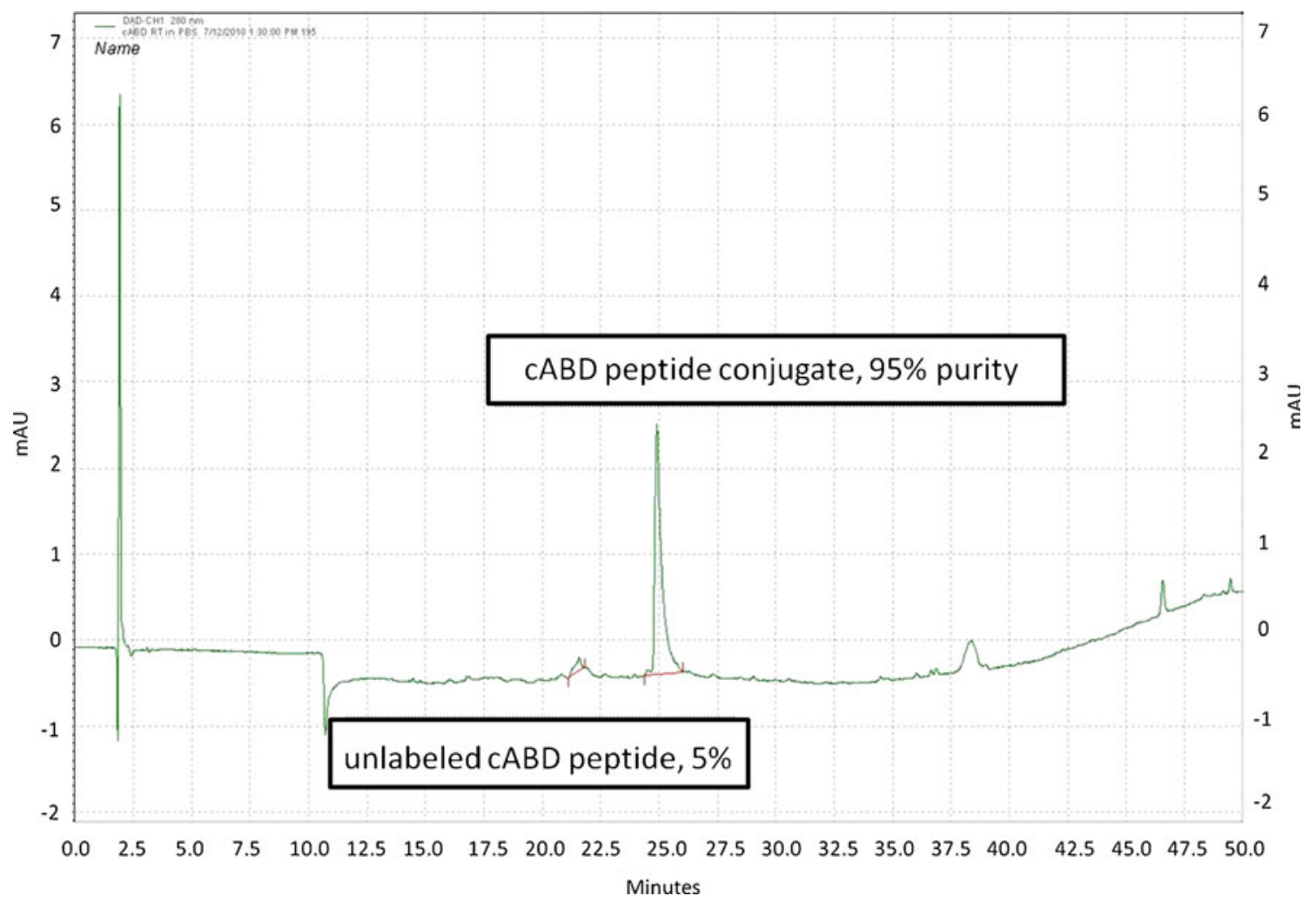

Fig. 2. HPLC chromatogram showing the cABD-IRDye800 peptide conjugate peak that was eluted from a Zorbax semi-prep C-18A column at 25 min with 95\% purity. The unconjugated (free) peptide and cABD-IRDye800 conjugate can be seen as separate peaks in the $280 \mathrm{~nm}$ channel.

previously described [40]. We coupled $\approx 900$ response units of MSA to a CM5 chip using standard NHS-EDC chemistry. A reference flow cell was inactivated for in-line subtraction. Kinetic analysis was performed at $25^{\circ} \mathrm{C}$ in HEPES-buffered saline EP+ buffer (GE Healthcare) at a flow rate of $60 \mu \mathrm{l} / \mathrm{min}$. Five 2-fold dilutions of each peptide beginning at $500 \mathrm{nM}$ were analyzed in duplicate, injected over the flow cells for $1 \mathrm{~min}$ and dissociation monitored for $5 \mathrm{~min}$. The surface was regenerated with glycine 3.0 and allowed to stabilize $30 \mathrm{~s}$ prior to subsequent injections. BIA evaluation software was used to determine Kd using a 1:1 binding model. Affinities provided were the average of three independent experiments for each peptide dilution series with standard deviation recorded.

To confirm binding specificity of the conjugate, a $0.2 \mathrm{mg} / \mathrm{mL}$ solution of cyclic peptide was incubated with an equimolar concentration of MSA or ovalbumin (OVA) or $5 \%$ human serum for $1 \mathrm{~h}$ at room temperature. The cyclic peptide conjugate, MSA, OVA, and free IRDye 800 were separated using sodium dodecyl sulfate-polyacrilamide gel electrophoresis (SDS-PAGE). Pre-cast 4-20\% long life gels (NuSep, Lawrenceville GA), were run using $1 \times$ Tris/Hepes/SDS running buffer for $1-1.5 \mathrm{~h}$ at $100 \mathrm{~V}$, followed by fluorescence imaging at $800 \mathrm{~nm}$ on an Odyssey NIR imager, at an intensity of 1.5 and focus offset of $3 \mathrm{~mm}$. Gels were then stained with Biosafe Coomassie Blue (BioRad, Hercules CA) overnight at $4^{\circ} \mathrm{C}$, to elucidate protein bands, and imaged using an Alpha Innotech gel imaging system (Cell Biosciences, Santa Clara CA). Similarly, to assess albumin binding of ICG and IRDye800, equimolar amounts of ICG and IRDye800, were each incubated with $0.2 \mathrm{mg} / \mathrm{mL}$ MSA or $5 \%$ human serum. SDS-PAGE was performed as described above, followed by fluorescence imaging at $800 \mathrm{~nm}$ using the Odyssey NIR imager. Gels were stained with coomassie blue overnight at $4^{\circ} \mathrm{C}$, to elucidate protein bands, and imaged using the Alpha Innotech gel imaging system.

\section{Measurement of Partition Coefficients}

The lipophilicity of cABD-IRDye 800 was assessed by determination of the water-octanol partition coefficient. 1-Octanol $(1 \mathrm{~mL})$ was added to a solution of approximately 40-50 $\mu \mathrm{g}$ of cABDIRDye 800 in water $(1 \mathrm{~mL})$, and the layers were vigorously vortexed for $5 \mathrm{~min}$ at room temperature and again mixed after $2 \mathrm{~h}$ for $3 \mathrm{~min}$. The tubes were centrifuged at $14,000 \mathrm{rpm}$ for $5 \mathrm{~min}$. Three samples of each layer were analyzed spectrophotometrically using the DU-800 Spectrophotometer (Beckman Coulter, Brea $\mathrm{CA})$, applying this formula:

$$
\text { Pconc. }(\mathrm{mg} / \mathrm{mL})=\left(\mathrm{A}_{280}-\left(0.03 \times \mathrm{A}_{780}\right) / \varepsilon_{\mathrm{P}}\right) \times \mathrm{MW}_{\mathrm{P}} \text {, }
$$$$
(\mathrm{P}=\mathrm{ICG} \text {, IRDye800 and cABD - IRDye800) }
$$

where $\mathrm{MW}$ is the molecular weight, $\varepsilon$ is the extinction coefficient, and $\mathrm{A}$ is absorption.

The partition coefficient was determined by calculating the ratio of concentration in octanol/concentration in water and expressed as $\log P$. At least three independent experiments were performed in triplicate to give the mean $\log P$ value. 


\section{In Vivo Studies}

All animal studies were approved by the University of Texas Health Science Center Houston, Center for Laboratory Animal Medicine and Care (CLAMC) animal welfare committee. Mice were housed in the university's AAALAC accredited facilities under sterile conditions according to CLAMC policy. Animal studies were conducted using shaved 6-7-week-old female, C57BL/6 mice (Harlan, Charles River). Mice were divided into experimental groups, anesthetized with isofluorane inhalation, 2$3 \%$ until unresponsive to painful stimuli and placed on a heating pad to maintain body temperature at $37^{\circ}$ during each imaging session ( $\sim 30 \mathrm{~min} /$ animal). Mice were closely monitored during and after imaging for any adverse events, of which there were none. Each mouse was injected intradermally at the base of the tail with $20 \mu \mathrm{L}$ of $500 \mu \mathrm{M} \mathrm{ICG}$, or $20 \mu \mathrm{L}$ of $100 \mu \mathrm{M}$ cABD-IRDye 800 , in order to assess the performance of each fluorophore in vivo. The injections were all made by the same investigator (C. D-V) and the injection site was covered with sterile tape immediately prior to imaging to block injection site fluorescence and prevent camera saturation. Figure 3 is a fluorescent image superimposed on a white-light image showing a typical view of normal murine lymphatic vasculature after injection of NIR fluorophore cABDIRDye 800 at the base of the mouse tail. Labels are included to orient the reader and highlight pertinent parameters including, injection site, a lymphatic vessel and inguinal lymph node. Animals were illuminated by a laser diode $(100 \mathrm{~mA}$ and $80 \mathrm{~mW}$ for $785 \mathrm{~nm}$, Sanyo; Richmond, IN) with the light source expanded to a uniform circular area approximately $8 \mathrm{~cm}$ in diameter. Fluorescence emission was collected using an EMCCD camera with a holo-

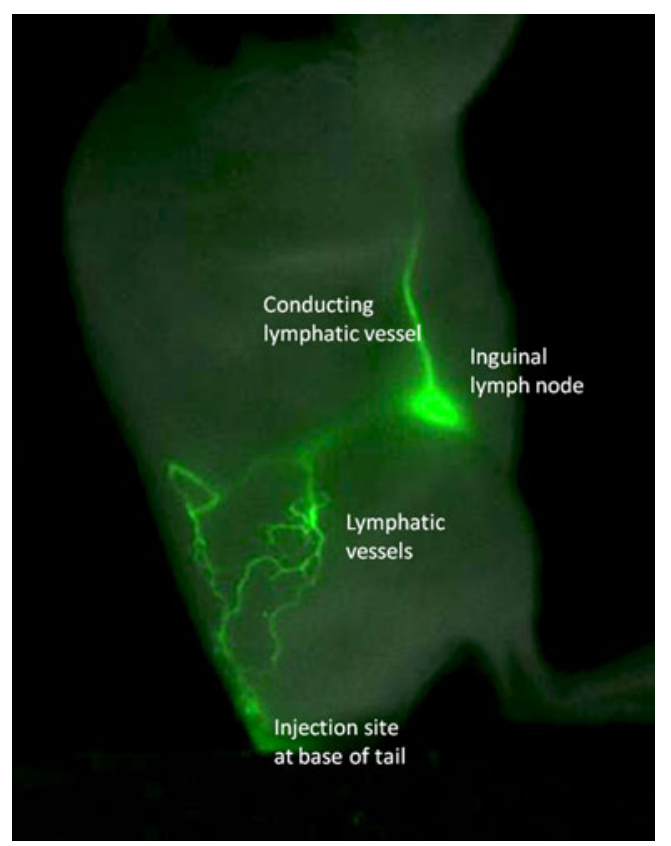

Fig. 3. An example of normal murine lymphatic vasculature as typically seen after injection of $20 \mu \mathrm{L}$ of $200 \mu \mathrm{M}$ cABDIRDye800 at the base of the tail, a near-infrared fluorescence image is overlaid on a white-light image to aid in reader orientation, pertinent parameters including injection site, lymph vessel, and inguinal lymph node are denoted. graphic notch-plus band-rejection filter (785-nm center wavelength for ICG) and a bandpass filter (830-nm center wavelength for ICG) placed prior to the lens to reject backscattered and reflected excitation light. Fluorescence images were acquired with an integration time of $200 \mathrm{~ms}$. For registration purposes, "white-light" images were taken by removing the filters and acquiring images over 500-ms integration time. Up to 750 fluorescent images and three white-light images were acquired immediately after injection on the left and right side of each animal. Statistical analyses of differences in fluorescent intensity between the agents at each time point were assessed by analysis of variance (ANOVA) and Student's $t$ test in which $\alpha=0.05$.

\section{Lymphatic Clearance}

C57/B16 mice were anesthetized using 2-3\% isoflurane during the induction phase and minimal $<1 \%$ isoflurane during the duration of the experiment which lasted $50 \mathrm{~min}$. Mice were maintained at $37^{\circ}$ using a heating pad or lamp. Anesthetized mice were intradermally injected with ICG $(n=5)$, IRDye800 $(n=4)$, and cABD-IRDye800, $(n=3)$, using a 31-gauge needle. To quantify lymphatic clearance of each agent, the fluorescence intensity within the region near the inguinal lymph node was recorded at five time points: 0.5, 2.5, 15, 30 , and 50 min after injection of each NIR agent. To account for differences in fluorescent intensity, $20 \mu \mathrm{L}$ of $500 \mu \mathrm{M}$ ICG or $20 \mu \mathrm{L}$ of $100 \mu \mathrm{M}$ of IRDye 800 and cABD-IRDye 800 were administered to each mouse and fluorescence images (200 ms/frame), were acquired immediately afterward and at each successive time point using the camera system described above. Seven hundred fifty images were taken for the first time point and 200 images for each successive time point. Images were processed using $\mathrm{V}++$ software (Digital Optics; Auckland, New Zealand), and data were analyzed to determine in vivo fluorescent intensities in select ROI using ImageJ (NIH, Bethesda, MD) and Matlab (The Mathworks). Fluorescence was normalized to the maximum fluorescence intensity within the inguinal lymph node for each agent and plotted as a function of time. Statistical analysis of the data were conducted using a Student's $t$ test with $\alpha=0.05$.

\section{Results}

\section{Characterization of Imaging Agents}

Table 2 lists the MW, extinction coefficient at $785 \mathrm{~nm}$, quantum yield at 785/830 (Ex/Em), and lifetime for $\mathrm{ICG}$, IRDye800CW, cABD-IRDye800 at 785/830 (Ex/Em). In addition, Table 2 also lists the brightness of IRDye 800 and cABD-IRDye800 relative to ICG which was computed by taking the ratio of $(($ extinction coefficient $) \times($ quantum yield $)) /$ (extinction coefficient of ICG) $\times($ quantum yield of ICG)). The results showed that IRDye800 itself was approximately five times brighter than ICG, and that on a molar basis the IRDye800 conjugate retained its brightness over ICG. cABDIRDye800 was 5.6 times brighter and had 1.6 times longer lifetime than ICG. In addition, the lifetime of IRDye800 and cABD-IRDye800 were similar to one another, indicating 
Table 2. Table of optical properties for NIR fluorophores

\begin{tabular}{lcclll}
\hline Agent & MW & Extinction coefficient ${ }^{\mathrm{a}}(\mathrm{M} \mathrm{cm})^{-1}$ & Quantum yield $^{\mathrm{b}}$ & Lifetime $^{\mathrm{b}}$ (ns) & Relative brightness vs. ICG \\
\hline ICG & 775 & $103,502 \pm 1.1 \mathrm{E} 4$ & $0.016^{\mathrm{c}}$ & $0.56^{\mathrm{c}}$ & 1 \\
IRDye800 & 1,166 & $178,378 \pm 7.1 \mathrm{E} 2$ & $0.046 \pm 3.12 \mathrm{E}-4$ & $0.93 \pm 0.075$ & 4.9 \\
cABD-IRDye800 & 3,401 & $171,025 \pm 1.4 \mathrm{E} 4$ & $0.054 \pm 9.28 \mathrm{E}-4$ & $0.92 \pm 0.102$ & 5.6 \\
\hline
\end{tabular}

\footnotetext{
${ }^{a}$ Absorbance, $\lambda=785 \mathrm{~nm}$

${ }^{\mathrm{b}}$ Excitation/emission, $\lambda=785 / 830 \mathrm{~nm}$

${ }^{\mathrm{c}}$ See Ref. [53]
}

IRDye800 is a consistent fluorophore when conjugated onto the cABD peptide.

Mass spectrometry confirmed that the deconvoluted molecular weight of the cABD-IRDye800 conjugate was 3,401 Da which was in agreement with the expected molecular weight of $3,404 \mathrm{Da}$ based on the sum of the known molecular weights of the cABD peptide and IRDye800. A subsequent HPLC stability study showed that the $\mathrm{CABD}$ peptide conjugate remained stable in $\mathrm{PBS}$ or water, at both room temperature and $4{ }^{\circ} \mathrm{C}$, through day 15 postconjugation. Percent purity of the conjugate immediately after HPLC purification, for each storage condition ranged between peak areas of $92-98 \%$. The conjugate peaks were maintained within a few percent of this initial area over the study period with the exception of water at room temperature for which one sample had a slightly decreased peak area of about $75 \%$.

\section{Affinity for Serum Protein}

The affinity of the conjugate for albumin was tested by comparing its $\mathrm{IC}_{50}$ and $\mathrm{Kd}$ for MSA to that reported by Dennis et al. for the peptide alone [40]. We found the purified cyclic peptide conjugate had an $\mathrm{IC}_{50}$ of $44.83 \pm 4.85 \mathrm{nM}$. Kd was assessed by surface plasmon resonance and the $\mathrm{Kd}$ of the unconjugated cABD peptide was found to be $65 \pm 10 \mathrm{nM}$. While measuring the $\mathrm{Kd}$ of cABD-IRDye800, interference between the $760 \mathrm{~nm}$ light source of the BIAcore and the peak excitation wavelength of IRDye 800 ( $\sim 774 \mathrm{~nm})$, possibly caused a recently described phenomenon known as surface plasmon-coupled emission resonance [54], which inverted the time response curve, and the $\mathrm{Kd}$ could not be determined. Therefore, the $\mathrm{Kd}$ of cABD conjugated to Dylight680, a spectrally and structurally similar near-infrared dye, was used to confirm conjugate binding to MSA. The $\mathrm{Kd}$ of cABD-Dylight680 was $32 \pm$ $5 \mathrm{nM}$. The sensograms of cABD-Dylight680 and cABDIRDye800 showed a similar pattern of binding indicating a constant angle of refraction with only changes due to reduction of signal intensity due to absorbance by the fluorophore.

\section{Sodium Dodecyl Sulfate-Polyacrilamide Gel Electrophoresis}

Binding specificity of peptide conjugate was assessed using SDS-PAGE. MSA was the positive control for peptide binding, while ovalbumin, a structurally distinct form of albumin was used as a negative control. The NIR fluorescence image in Fig. 4a shows that a fluorescent band was found only in the samples in which MSA or human serum (but not OVA) was incubated with cABD-IRDye800. This fluorescent band migrated to $\sim 70 \mathrm{kD}$, which is the approximate molecular weight of cABD-IRDye800 bound to albumin, thus confirming binding. Figure $4 \mathrm{~b}$ is the corresponding coomassie protein stained image of the gel which shows, the conjugate bound to MSA (70 kD), MSA (67 kD), and OVA (48 kD), at their expected molecular weights and shows the bands of the human serum proteins as well. The result that the cABD conjugate bound to MSA but did not bind to ovalbumin confirms the specificity of the interaction between the $\mathrm{CABD}$ peptide conjugate and MSA. Furthermore, the albumin binding capabilities of ICG and IRDye 800 were assessed by incubating both fluorophores in MSA or $10 \%$ human serum. ICG showed binding in both MSA, as was seen by the $\sim 68 \mathrm{kD}$ fluorescent band in Fig. 5a, and serum solution; while IRDye800 appeared to bind to MSA but not human serum. This result suggests that IRDye 800 may be binding too weakly to be detected in human serum. The corresponding protein image is shown in Fig. 5 b.

\section{Partition Coefficients}

In order to assess possible differences in hydrophobicity that may impact binding to albumin, the partition coefficients of ICG, IRDye 800 and cABD-IRDye 800 as determined from the ratio of each fluorophores' concentration in octanol and water were measured and expressed as $\log P$ values. There was a small but significant difference $(p<0.05$ by ANOVA), in the $\log P$ values between the fluorophores, with IRDye 800 having the smallest $\log P,-1.13+/-0.08$ indicating the greatest concentration of fluorophore in the aqueous phase followed by ICG, $-0.69+/-0.03$ and then cABD-IRDye800, $-0.53+/-0.005$.

\section{In Vivo Lymphatic Imaging Studies}

Figure 6 shows NIR fluorescence images of the dorsal lateral side of mice injected with $20 \mu \mathrm{L}$ of (a) $500 \mu \mathrm{M}$ ICG or (b) $100 \mu \mathrm{M}$ of cABD-IRDye 800 . Similarly to ICG, the peptide conjugate was visible in the lower trunk region as well as the 

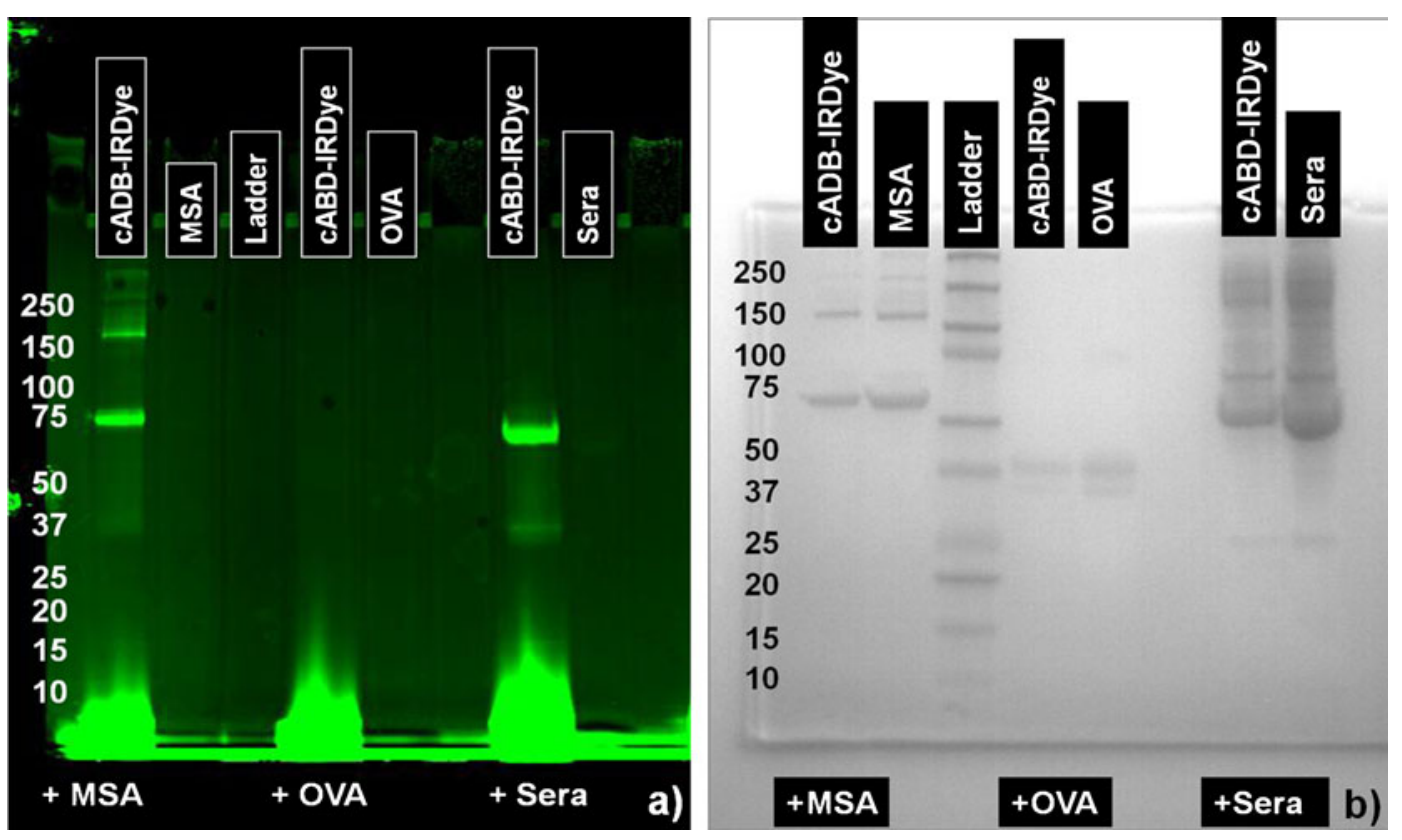

Fig. 4. SDS-PAGE shows $\mathbf{a}$ fluorescence and $\mathbf{b}$ protein images of CABD-IRDye800 after incubation with MSA or OVA, showing specificity of the conjugate for MSA.

inguinal lymph node and the collecting lymphatic vessels leading up to the axillary lymph node. These results indicate the conjugate is able to be taken up, traffic through the lymphatic vasculature and discern key lymphatic features at concentrations lower than that of ICG.

\section{Lymphatic Clearance Study}

The lymphatic clearance of ICG, IRDye800, and cABDIRDye800 from the inguinal lymph node was investigated by measuring the fluorescence intensities within the node at given intervals between 0 and $50 \mathrm{~min}$. Fluorescent intensities for each fluorophore were normalized to the maximal fluorescence intensity due to variations in absolute fluorescent counts measured within each region of interest surrounding the inguinal lymph node. An assessment of the percent of the maximal total fluorescence at each time point not only provided a measure of the clearance rate of the dye from the lymphatics, but also showed the relative rate of fluorophore entry and exit from the node. cABDIRDye800 entered the node more rapidly, as by $2.5 \mathrm{~min}$ the fluorophore had already reached its maximal fluorescence while ICG did not reach maximum until 15 min. Conversely,
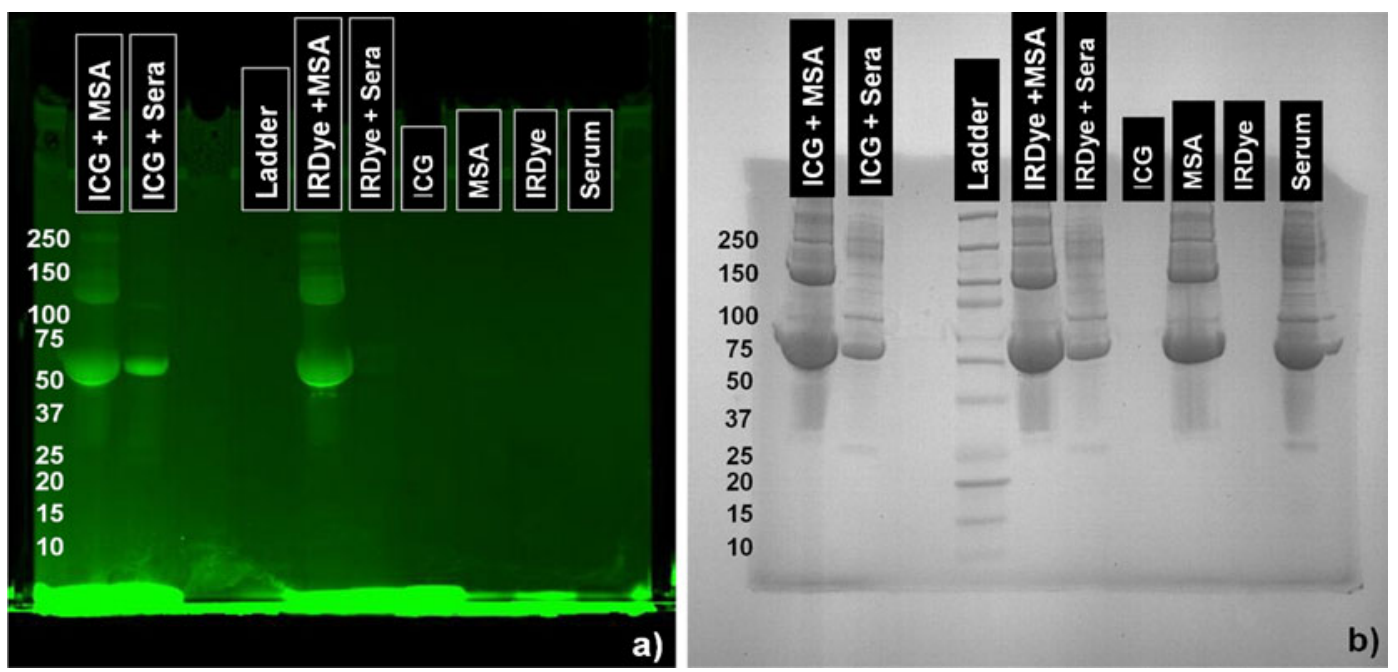

Fig. 5. SDS-PAGE shows $\mathbf{a}$ fluorescence and $\mathbf{b}$ protein images of ICG and IRDye800 after incubation with MSA and human serum, showing ICG binds to both, while IRDye800 may only bind MSA. 
the IRDye800 cleared rapidly, while ICG and cABDIRDye 800 retained at least $80 \%$ of the maximal fluorescence intensity over the study period. (Normalization is necessary because ICG is a dimmer fluorophore than IRDye800, and thus a higher concentration, $500 \mu \mathrm{M}$ (as determined from prior dose escalation studies) is used to visualize the lymph node. This increased fluorescence changes the fluorescent intensity as measured by ImageJ software. Thus, one cannot meaningfully compare the individual fluorescent intensities between each agent. Furthermore, the specific fluorescent intensities would not be able to be replicated by another user as they are specific to our unique instrumentation.) As the fluorophore cleared from the node there was an expected decrease in fluorescence intensity over time. Figure 7 shows comparisons of the lymphatic clearance of (a) ICG vs. IRDye 800 , (b) cABD-IRDye800 vs. IRDye800, and (c) ICG vs. cABD-IRDye800. ICG had a trend of higher fluorescence intensities than IRDye 800 at 15,30 , and $50 \mathrm{~min}$, although only the $30 \mathrm{~min}$ time point reached significance while cABD-IRDye 800 showed significantly higher fluorescence than IRDye 800 at 30 and $50 \mathrm{~min}$. ICG showed a trend similar to that of cABD-IRDye 800 but did not reach significance. Statistics were performed using ANOVA and Student's $t$ test with $\alpha=0.05$. The time points in this study were selected to determine initial baseline clearance data during an imaging session of approximately $50 \mathrm{~min}-1 \mathrm{~h}$, as is typical in human clinical trials. In the preclinical studies, the $\sim 1 \mathrm{~h}$ time frame allowed the anesthesia to be maintained at a low level over the study period as opposed to awakening and re-anesthetizing the animals. As it is clear from the communicated studies that IRDye 800 has a statistically significantly faster clearance than the other agents, the purpose of the study was met within this time frame. Additional lymphatic clearance studies and in vivo whole body clearance and pharmaco- kinetics over longer time points may be addressed in further studies.

\section{Discussion}

Due to its fluorescent properties, ICG has the ability to provide fluorescence contrast for vascular imaging. However, its low quantum efficiency and instability in solution [55] make ICG a poor fluorophore. Its chemical structure also limits its ability to be conjugated to targeting moieties. Consequently, other imaging agents including red excitable dyes such as Cy 5.5, and probe systems such as quantum dots have been pursued for imaging purposes, however, these agents may be limited by reduced tissue penetration, rapid vascular clearance, and/or questions on uncertain fate and toxicity. Herein, we have described the optical properties of cABD-IRDye800, a novel NIR peptide conjugate and its application in preclinical murine lymphatic imaging. In vitro studies confirmed the higher extinction coefficient and quantum efficiency of the IRDye 800 conjugate as compared with ICG, thus showing the enhanced absorptive and fluorescence capacity provided by IRDye800. Unlike the findings of other studies [56], we did not find that the extinction coefficient or quantum yield of $1 \mu \mathrm{M}$ ICG solution increased with the addition of albumin, although it is well known that at higher solution concentrations, ICG self-quenches and the addition of albumin or aspartic acid [57] can improve fluorescent yield of ICG. In addition, cABD-IRDye 800 was found to be significantly more stable than ICG and maintained integrity through a 15-day study period in water and PBS at room temperature and $4^{\circ} \mathrm{C}$. These results are in sharp contrast to ICG which begins to degrade within hours and at ambient temperature under normal light has a half life of only $14 \mathrm{~h}$ [27]. More importantly at the clinically relevant excitation wavelength
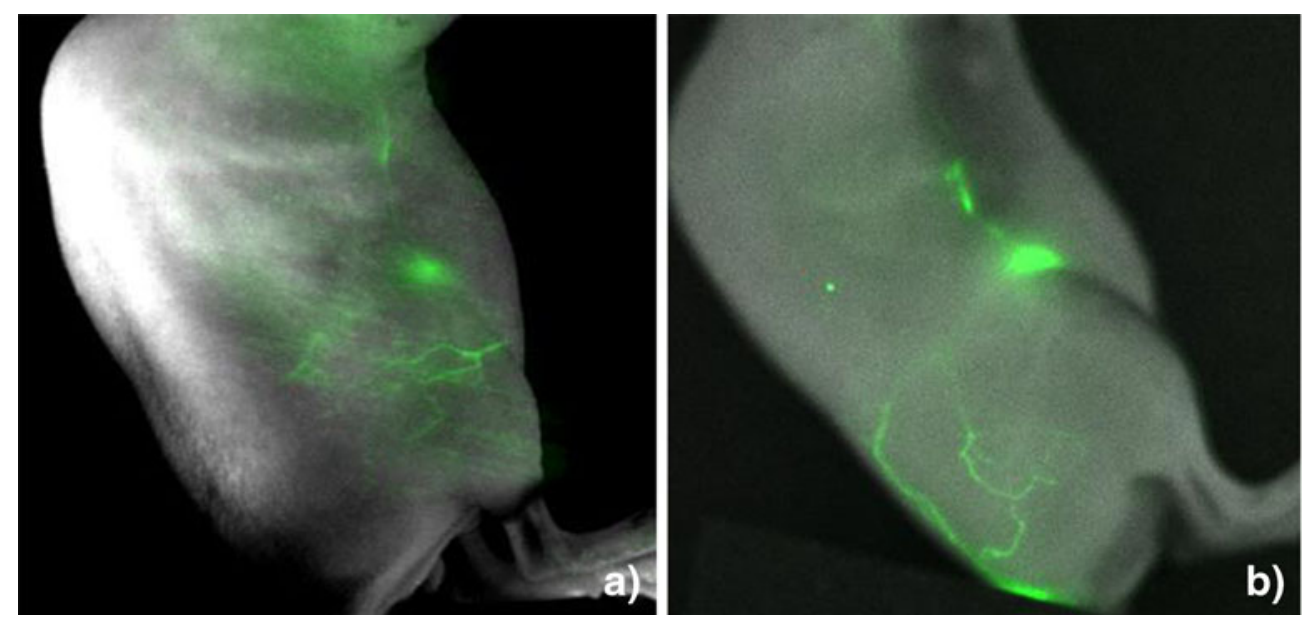

Fig. 6. Comparative near-infrared fluorescence imaging of normal $\mathrm{C} 57 \mathrm{BI} / 6$ mice injected at the base of the tail with $20 \mu \mathrm{L}$ of a $500 \mu \mathrm{M}$ ICG or b $100 \mu \mathrm{M}$ cABD-IRDye800, showing visualization of the initial superficial lymphatic vasculature, the inguinal lymph node and a conducting lymphatic vessel leading from the inguinal to axillary node. Imaging reveals ability of the peptide conjugate to provide comparable imaging quality at a lower dose than ICG. 

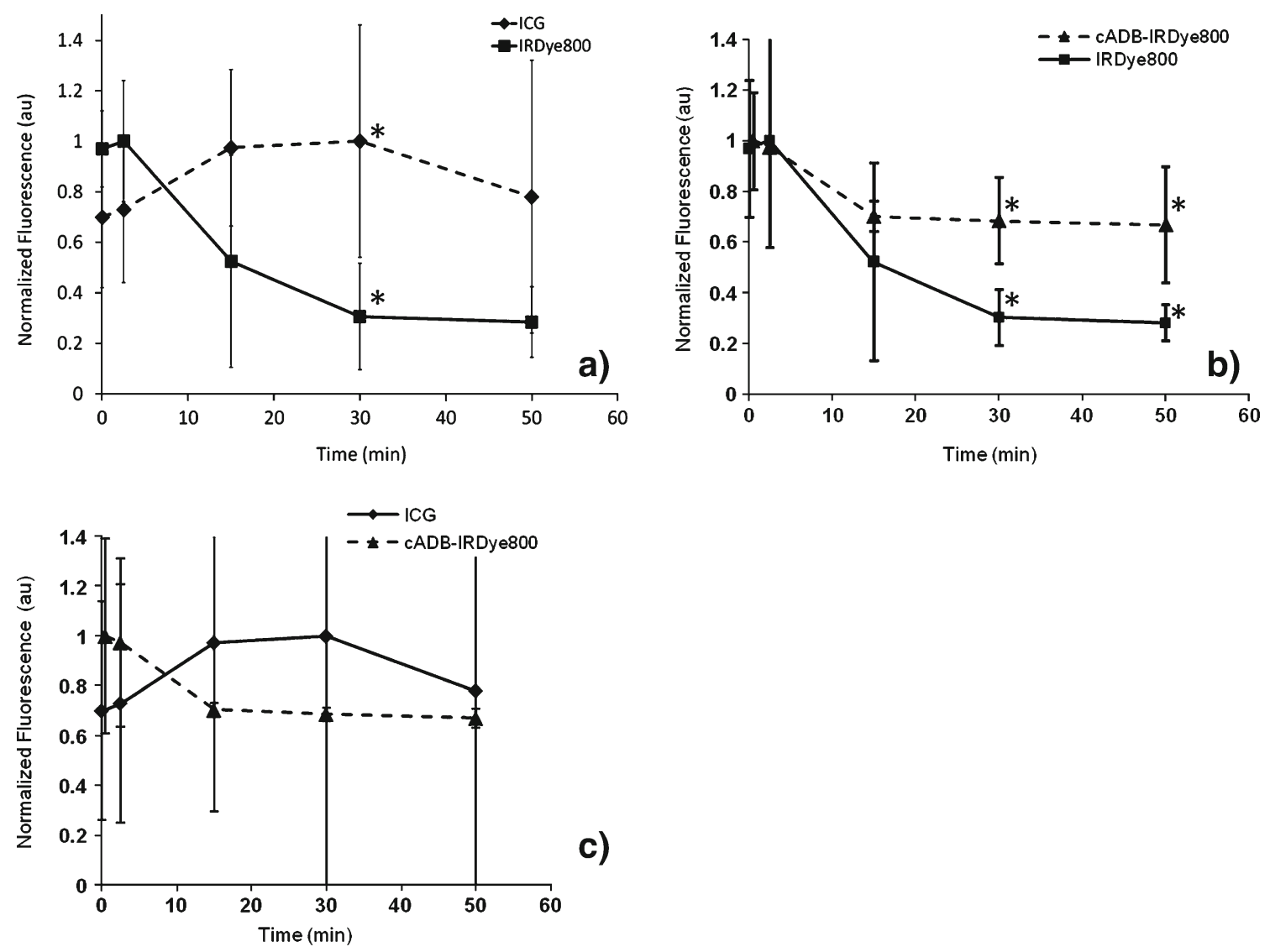

Fig. 7. Lymphatic Clearance Study: C57BI/6 mice were injected at the base of the tail with $20 \mu \mathrm{L}$ of $500 \mu \mathrm{M}$ ICG or $100 \mu \mathrm{M}$ IRDye800 or CABD-IRDye800 and imaged over several time points between 0 and 50 min to determine retention of fluorophores within the inguinal lymph node. Clearance from the node was compared between a ICG and IRDye800, b cABDIRDye800 and IRDye800, and c ICG vs. cABD-IRDye800.

( $\sim 785 \mathrm{~nm}$ ), ICG shows an even greater reduction in stability, thereby further reducing its useful lifetime. As such the brighter and more stable cABD-IRDye 800 provides an opportunity to improve upon these limitations. For further characterization of the peptide conjugate, binding assays with MSA were performed and showed that upon conjugation to IRDye800, the cABD peptide was able to maintain its specificity and affinity for albumin, thus confirming that the addition of the fluorophore did not significantly alter the binding properties of the peptide. The $\mathrm{IC}_{50}$ of $\mathrm{cABD}$ IRDye800 for MSA was found to compare favorably to the unlabeled cABD peptide as described by Dennis et al. [40]. In addition, the $\mathrm{Kd}$ of cABD-Dylight680 was in the nanomolar range again showing a high affinity of the conjugate for MSA, and consistency of the peptide once conjugated to a fluorophore. Although albumin-binding domain conjugates with higher binding affinity for albumin have been described $[45,50]$, a recent study has shown that increasing affinity beyond the nanomolar range has only minimal effects on increasing half life and that half-life extension by $\mathrm{ABD}$ is only weakly influenced by affinity for albumin or valency [47]. As such, we have selected the peptide conjugate that provides the optimal combination of favorable properties for binding to albumin, allowing for its retention in the lymphatics, as well as for in vivo lymphatic imaging.

The ability of the conjugate to bind albumin and serum proteins is significant because it allows for the imaging of protein movement from the tissue space into the lymphatics. This is relevant to the present study because while the blood circulation is capable of taking up fluid and small proteins, large proteins and macromolecules can only be taken up by the large openings in the lymphatic capillaries. Therefore, when the peptide conjugate associates with these large serum proteins, it is preferentially taken up by the lymphatics. The protein association could be especially useful in monitoring diseases such as lymphedema in which impaired lymphatic flow causes an accumulation of protein in the interstitial space followed by a rise in oncotic pressure and the consequent buildup of excess fluid in the tissue, causing edema. Thus, the conjugate affords us the unique opportunity to study a key aspect of the development of edema and to assess the role of protein movement in the progression of lymphatic disease.

Although ICG is also known to associate with serum proteins, it is difficult to find direct experimental evidence 
that confirms this result. Therefore, we conducted studies comparing the serum binding properties of both ICG and IRDye800. We found that as expected, ICG associates with both MSA and human serum showing similar results to that of cABD-IRDye800. Despite this finding, ICG has significant limitations which include its poor optical properties and instability in solution. Also of considerable importance for use in lymphatic imaging is the ability of the fluorophore to provide bright signal imaging when injected in microdose quantities. This has significant implications for the reconstitution and use of ICG in a clinical setting to discern the presence of lymphatics. In fact, when directly comparing the stability and fluorescence of ICG and IRDye800, the latter is clearly superior and serves as a good alternative to ICG. However, clearance studies show that IRDye800 rapidly clears from the lymphatics, likely due to a reduced association to serum proteins. Therefore, we have selected the cABD-IRDye 800 conjugate which allows us to maximize fluorescent yield and enhance lymphatic retention.

Interestingly, although IRDye800 did appear to associate with MSA, it did not appear to bind to human serum even though the molar concentrations and conditions for incubation were the same for ICG and IRDye800. These results indicate that IRDye800 may have a reduced affinity for or inability to bind human serum proteins and therefore may not be useful for imaging protein movement in clinical lymphatic studies.

To assess another factor that may impact binding to albumin, the partition coefficient of each fluorophore in octanol and water was measured, which provided an order of hydrophobicity for the fluorophores as cABD-IRDye800> ICG $>$ IRDye800. These results suggest that the slightly increased hydrophobicity of ICG and cABD-IRDye 800 may favor their binding within the hydrophobic albumin binding pocket as compared with IRDye 800 and may play some part in the increased retention time of these two agents within the lymphatics.

From these studies, there are several significant advantages to using cABD-IRDye800 for imaging lymphatics as compared with a free dye alone. First, the cABD peptide conjugate is uniquely directed to the lymphatic system due to its high affinity for albumin thereby enhancing vascular retention, as opposed to a free dye which may have a more transient interaction or be small enough to be taken up into the blood vasculature. Second, by conjugation to the bright near-infrared dye, IRDye 800, the imaging agent gains a brighter fluorescent intensity, enhanced optical properties, and long term stability in solution - all necessary for imaging key structures of the lymphatics. In support of our selection of this fluorophore conjugate, a previous study [56] showed that in comparison to several other NIR fluorophores including ICG, an IRDye800 conjugate of albumin had the best outcome with respect to physical properties, uptake and retention, fluorescence signal and reproducibility in sentinel lymph node mapping. Our albumin-binding domain conjugate has these favorable properties without direct conjugation at undefined sites to a bulky albumin molecule. Third, the peptide and dye are both well characterized, the conjugate is relatively small, readily synthesized and has a quantifiable 1:1 conjugation chemistry, all of which significantly enhance its ability to be used clinically.

Subsequent comparative murine imaging studies confirmed that cABD-IRDye 800 shows uptake and retention in the lymphatics, as well as high sensitivity imaging at lower concentrations than ICG. These results again highlight the superior optical properties of the conjugate. The ability to use a brighter NIR fluorophore for imaging allows for the delivery of microdose quantities of the imaging agent, which not only reduces the possibility for any adverse reactions but also reduces the stringency of FDA criteria in subsequent clinical studies, thus allowing more rapid advancement of the imaging agent to the clinic. As we intend to employ NIR fluorophores for clinical use, it is also important that the fluorophore be able to remain in the lymphatics for at least $1 \mathrm{~h}$ during the patient imaging session, which was reflected in the time points chosen for the clearance study. Our clearance study indicated that cABD-IRDye 800 and ICG were both retained in the lymph node longer than IRDye 800 as determined by the comparatively higher total fluorescence in the lymph node. IRDye800 (as a free dye), was able to rapidly enter the lymphatics, but also appeared to clear very rapidly from the inguinal lymph node and had less than $30 \%$ of maximum fluorescence remaining at $50 \mathrm{~min}$. The more rapid clearance of IRDye 800 may be attributed to the fact that unlike ICG, which associates with albumin and serum proteins [58] that may aid in lymphatic retention, IRDye 800 may have a more transient or weaker association with MSA, and may not bind human serum proteins at a detectable level. This result is substantiated by the similar lymph node clearance behavior of ICG and cABD-IRDye800, which differed from that of IRDye 800 alone. Thus, IRDye 800 may not reflect physiological protein movement in the lymphatics as do ICG and cABD-IRDye 800 and may clear too rapidly to be useful. In particular, when imaging lymphedema subjects with NIR fluorescence, it may not be warranted to intradermally inject additional fluid volume or protein into fibrotic tissue; therefore, retention of the initial microdose over the duration of the imaging session is important. Several fluorophores have been directly conjugated onto large proteins or molecules such as albumin, polyethylene glycol or hyaluronan to achieve this purpose; however, the large size of these conjugates as well as potential issues with chemistry, manufacturing and control of a large molecule, may limit their clinical applicability. As a consequence, we seek to intradermally administer the small, labeled cABD peptide for in situ binding of serum protein and subsequent lymphatic transit, in order to enable a diagnostic measure of protein movement. In addition, safety and toxicity studies with IRDye 800 show a favorable toxicity profile [51], and studies of similar ABD peptides in murine models also suggest safety and efficacy [40], thus we expect future studies will confirm similar outcomes for the conjugate. We anticipate this technology will be useful in addressing the cause of patient 
edema, assessing treatment strategies, and evaluating specific changes in the vasculature over time. In addition, Kwon and Sevick-Muraca [59] used ICG in preclinical animal models of cancer metastasis to demonstrate progressive changes in lymphatic vascular structure and transport function with advancing disease. A brighter, cABD-based agent may also be useful to better image tumor lymphangiogenesis in cancer patients. Since several emerging therapeutics against tumor lymphangiogenesis are under development, a more effective agent could be used to stratify patients who could be candidates for anti-lymphangiogenesis therapy as well as provide a metric to evaluate therapeutic response.

\section{Conclusions}

Based upon our collective results, cABD-IRDye800 appears to be a favorable imaging agent for lymphatic imaging studies. cABD-IRDye800 provides a unique opportunity to noninvasively image the lymphatics and to enhance our understanding of the underlying pathology of lymphatic disease for improved patient diagnosis, monitoring, and treatment. The ability to translate our imaging agent, and other subsequent vascular imaging agents into the clinic is of utmost importance as it has significant implications for improving disease management and outcome in a patient population in which there is very limited knowledge of disease pathogenesis and few treatment options. Taken together, our peptide conjugate and noninvasive NIR imaging technology have the ability to be an effective tool to begin to address the challenges of diagnosing and treating lymphatic disease. Elucidating the changes that occur in lymphatic disease will help us to further understand the complex interplay that occurs between the lymphatics, blood vasculature, and other organ systems in vascular disease, and may lead to future discoveries in related metabolic disorders such as diabetes, obesity, and cancer.

Acknowledgments. This work was supported in parts by R01 CA112679-15 (supplement to C. D-V), R01 CA128919-03 (EMS-M), R01 HL09293-03 (EMS-M), and the Howard Hughes Medical Institute Med into grad initiative (to C.D-V).

Conflict of interest. The authors declare that there are no conflicts of interest.

Open Access. This article is distributed under the terms of the Creative Commons Attribution Noncommercial License which permits any noncommercial use, distribution, and reproduction in any medium, provided the original author(s) and source are credited.

\section{References}

1. Rockson SG (2001) Lymphedema. Am J Med 110:288-295

2. Alitalo K, Tammela T, Petrova TV (2005) Lymphangiogenesis in development and human disease. Nature 438:946-953

3. Ferrell RE, Levinson KL, Esman JH et al (1998) Hereditary lymphedema: evidence for linkage and genetic heterogeneity. Hum Mol Genet 7:2073-8

4. Irrthum A, Karkkainen MJ, Devriendt K, Alitalo K, Vikkula M (2000) Congenital hereditary lymphedema caused by a mutation that inactivates VEGFR3 tyrosine kinase. Am J Hum Genet 67:295-301

5. Irrthum A, Devriendt K, Chitayat D et al (2003) Mutations in the transcription factor gene SOX18 underlie recessive and dominant forms of hypotrichosis-lymphedema-telangiectasia. Am J Hum Genet 72:1470-8

6. Fang J, Dagenais SL, Erickson RP et al (2000) Mutations in FOXC2 (MFH-1), a forkhead transcription factor are responsible for hereditary lymphedema-distichiasis syndrome. Am J Hum Genet 67:1382-1388

7. Lyon MF, Glenister PH (1984) Mouse News Lett 71:26

8. Karkkainen MJ, Saaristo A, Jussila L et al (2001) Model for gene therapy for human hereditary lymphedema. PNAS 23:12677-82

9. Francois M, Caprini A, Hosking B et al (2008) Sox18 induces development of lymphatic vasculature in mice. Nature 456:643-7

10. Kriederman BM, Myloyde TL, Witte MH et al (2003) FOXC2 haploinsufficient mice are a model for human autosomal dominant lymphedema-distichiasis syndrome. Hum Mol Genet 10:1179-1185

11. Finegold DN, Schacht V, Kimak MA et al (2008) HGF and MET mutations in primary and secondary lymphedema. Lymphat Res Biol 6:65-8

12. Sharma R, Wendt JA, Rasmussen JC, Adams KE, Marshall MV, Sevick-Muraca EM (2008) New horizons for imaging lymphatic function. Ann NY Acad Sci 1131:13-36

13. Szuba A, Shin WS, Strauss HW, Rockson S (2003) The third circulation: radionuclide scintigraphy in the evaluation of lymphedema. J Nucl Med 44:43-57

14. Yuan Z, Chen L, Luo Q, Zhu J, Lu H, Zhu R (2006) The role of radionuclide lymphoscintigraphy in extremity lymphedema. Ann Nucl Med 20:341-344

15. Witte CL, Witte MH, Unger EC et al (2000) Advances in imaging of lymph flow disorders. Radiographics 20:1697-719

16. Marotel M, Cluzan R, Ghabboun S, Pascot M, Alliot F, Lasry JL (1998) Transaxial computer tomography of lower extremity lymphedema. Lymphology 31:180-85

17. Lohrmann C, Foeldi E, Langer M (2009) MR imaging of the lymphatic system in patients with lipedema and lipo-lymphedema. Microvasc Res 77:335-9

18. Marshall MV, Rasmussen JC, Tan I-C et al (2010) Near-infrared fluorescence imaging in humans with indocyanine green: a review and update. Open Surg Oncol J 2:12-25

19. Craandijk A, Van Beek CA (1976) Indocyanine green fluorescence angiography of the choroid. Brit J Opthal 60:377-85

20. Cherrick GR, Stein SW, Leevy CM, Davidson CS (1960) Indocyanine Green: observations on its physical properties, plasma decay and hepatic extraction. J Clin Investig 39:592-600

21. Sevick-Muraca, EM, Sharma R, Rasmussen JC et al. (2008) Imaging of lymph flow in breast cancer patients after microdose administration of a near-infrared fluorophore. Radiology 246:734-741

22. Rasmussen JC, Tan I-C, Marshall MV, Fife CE, Sevick-Muraca EM (2009) Lymphatic imaging in humans with near-infrared fluorescence. Curr Opin Biotechnol 20:1-9

23. Maus EA, Tan I-C, Rasmussen JC et al. (2010) Near-infrared fluorescence lymphatic imaging of head and neck lymphedema. Head and Neck PMID: 21077150

24. Adams KE, Rasmussen JC, Darne C et al (2010) A method to assess lymphatic response to advanced pneumatic compression device treatment of lymphedema. Biomed Opt Express 1:114-125

25. Rasmussen JC, Tan I-C, Maus EA et al (2010) Human lymphatic architecture and dynamic transport imaged using near-infrared fluorescence. Transl Oncol 3:362-72

26. Tan I-C, Maus EA, Rasmussen JC et al (2011) NIR fluorescence imaging of improved lymphatic propulsion and transport following manual lymphatic drainage. Arch Phys Med Rehabil (in press)

27. Saxena V, Sadoqi M, Shao J (2003) Degradation kinetics of indocyanine green in aqueous solution. J Pharm Sci 92:2090-97

28. Philip R, Penzkofer A, Baumler W, Szeimies RM, Abels C (1996) Absorption and fluorescence spectroscopic investigation of indocyanine green. J Photochem Photobiol A 96:137-38

29. Landsmen ML, Kwant G, Mook GA, Zijlstra WG (1976) Lightabsorbing properties, stability and spectral stabilization of Indocyanine green. J Appl Physiol 40:575-583

30. Fiehn C, Kratz F, Sass G, Muller-Ladner U, Neumann E (2008) Targeted drug delivery by in vivo coupling to endogenous albumin: an albumin-binding prodrug of methotrexate (MTX) is better than MTX in the treatment of murine collagen-induced arthritis. Ann Rheum Dis 67:1188-91

31. Xie D, Yao C, Wang L et al (2010) An albumin conjugated peptide exhibits potent anti-HIV activity and long in vivo half- life. Antimicrob Agents Chemother 54:191-6 
32. Chen K, Xie J, Chen X (2009) RGD-human serum albumin conjugates as efficient tumor targeting probes. Mol Imaging 8:65-73

33. Prakash J, Bansal R, Post E, de Jagger-Krikken A, Lub de Hooge MN, Poelstra K (2009) Albumin binding and tumor vasculature determine the anti-tumor effect of 15 -deoxy $\Delta^{12,14}$ prostaglandin-J2 in vivo. Neoplasia 11:1348-58

34. Lo et al (2011) Targeted human serum albumin nanoparticles for uptake in EGFR colon carcinoma. Nanomedicine (in press)

35. Dubey PK, Singodia D, Verma RK, Vyas SP (2011) RGD modified albumin nanospheres for tumor vasculature targeting. J Pharm Pharmacol 63:33-40

36. Anhorn MG, Wagner S, Kreuter J, Langer K, von Briesen H (2008) Specific targetting of HER2 overexpressing breast cancer cells with human serum albumin nanoparticles. Biconjugate Chem 19:2321-31

37. Kratz F, Mueller-Driver R, Hofmann I, Drevs J, Unger C (2000) A novel macromolecular prodrug concept exploiting endogenous serum albumin as a drug carrier for cancer chemotherapy. J Med Chem 43:1253-1256

38. Linhult M, Binz HK, Uhlén M, Hober S (2002) Mutational analysis of interaction between albumin binding domain from streptococcal $\mathrm{G}$ protein and serum albumin. Protein Sci 11:206-213

39. Johansson (2002) Structure, specificity and mode of action for bacterial albumin binding modules. J Biol Chem 277:8114-20

40. Dennis M, Zhang M, Meng YG (2002) Albumin binding as a general strategy for improving the pharmacokinetics of proteins. J Biol Chem 277:35035-43

41. Nguyen A, Reyes A, Zhang M et al (2006) The pharmacokinetics of an albumin-binding Fab (AB.Fab) can be modulated as a function of albumin binding. Protein Eng Des Sel 19:291-297

42. Stork R, Muller E, Kontermann RE (2007) A novel tri-functional antibody fusion protein with improved pharmacokinetic properties generated by fusing a bispecific single-chain diabody with an albumin binding domain from Streptococcal protein G. Protein Eng Des Sel 20:569-76

43. Müller D, Karle A, Meissburger B, Höfig I, Stork R, Kontermann RE (2007) Improved pharmacokinetics of recombinant bispecific antibody molecules by fusion to human serum albumin. JBC 282:12650-60

44. Anhorn MG, Wagner S, Kreuter J, Langer K, von Briesen H (2008) Specific targeting of HER2 overexpressing breast cancer cells with doxorubicin-loaded, trastuzumab modified human serum albumin nanoparticles. Bioconjug Chem 19:2321-31

45. Jonsson A, Dogan J, Herne N, Abrahmsén L, Nygren PA (2008) Engineering of a femtomolar affinity binding protein to human serum albumin. Prot Eng Des Sel 21:515-27
46. Trussel S, Dumelin C, Frey K, Villa A, Buller F, Neri D (2009) New strategy for the extension of the half-life of antibody fragments. Bioconjug Chem 20:2286-92

47. Hopp J, Hornig N, Zettlitz KA et al (2010) Effects of affinity and valency of albumin binding domain on half life. Protein Eng Des Sel 23:827-34

48. Sebak S, Mirzaei M, Malhotra M, Kulamarva A, Prakash S (2010) Human serum albumin nanoparticles as an efficient noscapine drug delivery system for potential use in breast cancer: preparation and in vitro analysis. Int $\mathrm{J}$ Nanomedicine 5:525-32

49. Vandoorne K, Addadi Y, Neeman M (2010) Visualizing vascular permeability and lymphatic drainage using labeled serum albumin. Angiogenesis 13:75-85

50. Andersen JT, Pehrson R, Tolmachev V, Daba MB, Abrahmsén L, Ekblad C (2011) Extending half-life by indirect targeting of the neonatal Fc receptor $(\mathrm{FcRN})$ using a minimal albumin binding domain. J Biol Chem 286:5234-41

51. Marshall MV, Draney D, Sevick-Muraca EM, Olive DM (2010) Single dose toxicity study of IRDye $800 \mathrm{CW}$ in Spraque-Dawley rats. Mol Imaging Biol 12:583-94

52. Williams ATR, Winfield SA, Miller JN (1983) Relative fluorescence quantum yields using a computer controlled luminescence spectrometer. Analyst 108:1067

53. Sevick-Muraca EM, Lopez G, Reynolds JS, Troy TL, Hutchinson CL (1997) Fluorescence and absorption contrast mechanisms for biomedical optical imaging using frequency domain techniques. Photochem Photobiol 66:55-64

54. Yuk JS, Trnavsky M, McDonagh C, MacCraith B (2010) Surface plasmon-coupled emission (SPCE)-based immunoassay using a novel paraboloid array biochip. Biosens Bioelectron 25:1344-49

55. Holzer W, Mauerer M, Penzkofer A et al (1998) Photostability and thermal stability of indocyanine green. J Photochem Photobiol B 47:155-64

56. Ohnishi S, Lomnes SJ, Laurence RG, Gogbashian A, Mariani G, Frangioni JV (2005) Organic alternatives to quantum dots for intraoperative near-infrared fluorescent sentinel lymph node mapping. Mol Imaging 4:172-181

57. Maarek JM, Holschneider DP, Harimoto J (2000) Fluorescence of indocyanine green in blood: intensity dependence on concentration and stabilization with sodium polyaspartate. J Photochem Photobiol B 65:157-64

58. Ott P, Weisiger RA (1997) Nontraditional effects of protein binding and hematocrit on uptake of indocyanine green by perfused rat liver. Am J Physiol 273:G227-38

59. Kwon S, Sevick-Muraca EM (2010) Functional lymphatic imaging in tumor-bearing mice. J Immunol Meth 360(1-2):167-172 